\title{
Microbial Characterization of Internal Active Thermal Control System (IATCS) Hardware Surfaces after Five Years of Operation in the International Space Station
}

\author{
Monsi C. Roman \\ NASA/ Marshall Space Flight Center \\ Natalee E. Weir and Mark E. Wilson \\ The Boeing Company \\ Barry H. Pyle \\ Montana State University
}

\begin{abstract}
A flex hose assembly containing aqueous coolant from the International Space Station (ISS) Internal Active Thermal Control System (IATCS) consisting of a 2 foot section of Teflon hose and quick disconnects (QDs) and a Special Performance Checkout Unit (SPCU) heat exchanger containing separate channels of IATCS coolant and iodinated water used to cool spacesuits and Extravehicular Mobility Units (EMUs) were returned for destructive analyses on Shuttle return to flight mission STS-114. The original aqueous IATCS coolant used in Node 1, the Laboratory Module, and the Airlock consisted of water, borate ( $\mathrm{pH}$ buffer), phosphate (corrosion control), and silver sulfate (microbiological control) at a pH of $9.5 \pm 0.5$. Chemical changes occurred after on-orbit implementation including a decrease to $\mathrm{pH} 8.4$ due to the diffusion of carbon dioxide through the Teflon hoses, an increase in nickel ions due to general corrosion of heat exchanger braze coatings, a decrease in phosphate concentration due to precipitation of nickel phosphate, and the rapid disappearance of silver ions due to deposition on hardware surfaces. Also associated with the coolant chemistry changes was an increase in planktonic microorganisms from less than 100 colony forming units (CFU) per $100 \mathrm{ml}$ to approximately 1 million CFU per $100 \mathrm{ml}$. Attachment and growth of microorganisms to the system surfaces (biofilm) was suspected due to the levels of planktonic microorganisms in the coolant. Biofilms can reduce coolant flow, reduce heat transfer, amplify degradation of system materials initiated by chemical corrosion, and enhance mineral scale formation.
\end{abstract}

Enumerations of microorganisms on hardware surfaces were performed by membrane filtration and spread plating on R2A, 1/10-strength tryptic soy agar, and fluid thioglycollate medium agar incubated for 14 days at 25 $30^{\circ} \mathrm{C}$. Enrichments of viable microorganisms from surfaces of the flex hose and SPCU heat exchanger were performed in R2B (R2A without agar), 1/10strength tryptic soy broth, and fluid thioglycollate medium. Bacterial colonies isolated from these assays were isolated and identified by several methods.

This initial paper reports the results of the membrane filtration analyses performed to assess biofilm on IATCS hardware exposed to coolant and microorganisms during five years of on-orbit operation.

\section{INTRODUCTION}

The heat generated by equipment and crew on board the International Space Station (ISS) is removed to maintain a comfortable working and living environment. The Thermal Control System (TCS) in the ISS collects excess heat directly from equipment and indirectly from the atmosphere. The TCS consists of two distinct sections: the Internal Active Thermal Control System (IATCS) which uses an aqueous solution as the working fluid to remove heat from the crew and equipment, and the External Active Thermal Control (EATCS) which uses ammonia as the working fluid to release heat to space via radiation. These two sections interact through liquid-liquid heat exchangers $(\mathrm{HX})$ that transfer heat while maintaining physical separation of the different fluids.

The IATCS consists of two loops that can be independently operated as a low-temperature loop and a moderate-temperature loop. These loops can also be operated in "single-loop mode" using the loop-crossover 
assembly, while maintaining their respective temperature ranges. A series of corrugated Teflon hoses and stainless steel tubing connects and transports the IATCS fluid from rack to rack and from the racks to the $\mathrm{HX}$ inside the ISS.

The IATCS fluid is also used to cool the spacesuits of the Extravehicular Mobility Units (EMUs). The HX that interfaces with the IACTS fluid and the EMU fluid is called the Special Performance Checkout Unit (SPCU). One side of the SPCU HX contains IATCS fluid; the EMU side contains iodinated water. Hamilton Sundstrand manufactures the IATCS HX and Honeywell manufactures the SPCU HX. The nickel braze material used in these heat exchangers are different. The IATCS $\mathrm{HX}$ contains BNi-2 material whereas the SPCU $\mathrm{HX}$ contains BNi-3.

\section{MICROBIOLOGICAL ENUMERATION AND ISOLATE IDENTIFICATIONS}

Coolant samples were collected aseptically in sterile containers containing $0.3 \mathrm{ml}$ of a $15 \% \mathrm{w} / \mathrm{v}$ solution of ethylenediaminetetraacetic acid (EDTA) per $100 \mathrm{ml}$ to neutralize potential inhibitory effects of nickel on the growth of microorganisms (1). Surface microbial samples were collected onto moistened sterile swabs and placed into $2.5 \mathrm{ml}$ of Neutralizing Buffer containing EDTA. Microorganisms were dispersed from the swab into the neutralizing buffer with sonication on ice 3 times for 30 seconds.

Viable plate counts were performed to enumerate microorganisms by standard membrane filtration (1). Serial dilutions of coolant and swab samples were filtered onto $0.45 \mu \mathrm{m}$ HAWG membrane filters (Millipore, Bedford, MA) that were $47 \mathrm{~mm}$ in diameter. The filters were transferred to $60 \mathrm{~mm}$ Petri dishes containing R2A, a growth medium designed to culture heterotrophic bacteria from low nutrient aqueous environments. The cultures were incubated at $30^{\circ} \mathrm{C}$ for 7 days, then $25^{\circ} \mathrm{C}$ for an additional 7 days. Colonies were counted at day 7 and day 14.

A minimum of $10 \%$ of the colonies for each different morphology were isolated into pure cultures for identification. This approach of identifying representative fractions of all the different colony morphologies on each plate provided increased probability of isolating all species and quantifying species predominance as compared to random sampling.

Identifications were performed with three automated identification systems including the Microbial Identification System (MIDI) fatty acid methyl ester gas chromatography, Biolog substrate utilization identification system, and the RiboPrinter ${ }^{\circledR}$ System that provides an automated genetic snapshot of any bacterial strain. Results from these automated systems were correlated with other published identifying characteristics for colony and cellular morphology, and individual biochemical tests such as nitrate reduction were performed for additional verification.

The MIDI system consists of a HP 6890 gas chromatograph equipped with a flame ionization detector, high resolution fused silica capillary column, split injection, and a robotic arm autosampler for unattended operation. Fatty acid methyl ester chromatographic peaks are automatically named, integrated, and compared to computerized reference libraries of known microorganisms by using the MIDI Sherlock Chromatography Pattern Recognition Software Version 3.1 and library upgrade 5.0. A statistical technique, cluster analysis, is used to generate a similarity index to organisms from the reference libraries (3).

The Biolog system consists of microtiter plates with 96 substrate assimilation tests, a computerized microtiter plate reader, Biolog Microlog 3 release 4.2 software and Gram positive and Gram negative bacterial reference libraries. Utilization of a substrate by bacteria causes the microtiter well to develop a blue color after a 4 or 24 hour incubation period. The reactions are automatically read and compared to the reference libraries. Cluster analysis similar to MIDI is used to generate a similarity index to organisms in the reference library (4).

The RiboPrinter ${ }^{\circledR}$ System is an automated ribosomal deoxyribonucleic acid (DNA) analyzer that lyses the cells and cuts the released bacterial DNA into fragments with a restriction enzyme (EcoRI or Pvull). The fragments are separated by size through gel electrophoresis, transferred to a membrane and hybridized with a DNA probe and mixed with a chemiluminescent agent. A digital camera captures the light emission as an image and the RiboPrinter ${ }^{\circledR}$ software (version 2.1) compares the pattern to a database of known library strains and determines similarity based on cluster analysis (5).

Microbiological enumeration results are reported as colony forming units (CFU) per unit volume for coolant samples or unit area for surface samples since colonies may arise from one or more cells. Coolant removed from the Flex Hose (sample FH002) contained $6.3 \times 10^{5}$ CFU/100 $\mathrm{ml}$ of bacteria (7-day incubation). Flex Hose 14-day biofilm counts were $12 \mathrm{CFU} / \mathrm{cm}^{2}$ on the inlet side (FH024, 1.5-2.5" long), $4 \mathrm{CFU} / \mathrm{cm}^{2}$ for a section midway along the tubing ( $\mathrm{FHO28}, 1.25-2.25^{\prime \prime}$ long), and 9 $\mathrm{CFU} / \mathrm{cm}^{2}$ at the outlet end (FH036, 1.5-2.5" long).

The isolate identifications for coolant removed from the flex hose and the inner surfaces of the flex hose are provided in Table 1. The CFU per unit volume or area 
are provided for each isolate to highlight species predominance in each of the sampled areas.

Table 1 Isolate Identifications for Flex Hose Coolant and Flex Hose Surfaces

\begin{tabular}{|c|c|c|c|}
\hline $\begin{array}{c}\text { Sample } \\
\text { Descriptio } \\
n\end{array}$ & Results & $\begin{array}{l}\text { Unit of } \\
\text { Measure }\end{array}$ & $\begin{array}{c}\text { Species } \\
\text { Identification }\end{array}$ \\
\hline $\begin{array}{l}\text { FH002 - } \\
\text { Fluid }\end{array}$ & $6.10 E+05$ & $\begin{array}{l}\text { CFU/ } \\
100 \mathrm{ml}\end{array}$ & $\begin{array}{l}\text { Ralstonia } \\
\text { eutrophalpaucula } \\
\text { (nit }+ \text { ) }\end{array}$ \\
\hline $\begin{array}{l}\text { FH002 - } \\
\text { Fluid }\end{array}$ & $2.00 E+04$ & $\begin{array}{l}\text { CFU/ } \\
100 \mathrm{ml}\end{array}$ & $\begin{array}{l}\text { Acidovorax } \\
\text { species (nit +) }\end{array}$ \\
\hline $\begin{array}{l}\text { FHO24- } \\
\text { Flex hose } \\
\text { Inlet }\end{array}$ & 5 & $\mathrm{CFU} / \mathrm{cm}^{2}$ & $\begin{array}{l}\text { Ralstonia } \\
\text { eutrophalpaucula } \\
\text { (nit -) }\end{array}$ \\
\hline $\begin{array}{l}\text { FHO24- } \\
\text { Flex hose } \\
\text { Inlet }\end{array}$ & 3 & $\mathrm{CFU} / \mathrm{cm}^{2}$ & $\begin{array}{l}\text { Unidentified non- } \\
\text { fermenting Gram } \\
\text { negative rod } \\
\text { (GNR) (nit -) }\end{array}$ \\
\hline $\begin{array}{l}\text { FH024- } \\
\text { Flex hose } \\
\text { Inlet }\end{array}$ & 2 & $\mathrm{CFU} / \mathrm{cm}^{2}$ & $\begin{array}{l}\text { Ralstonia } \\
\text { eutrophalpaucula } \\
\text { (nit +) }\end{array}$ \\
\hline $\begin{array}{l}\text { FH024- } \\
\text { Flex hose } \\
\text { Inlet }\end{array}$ & 2 & $\mathrm{CFU} / \mathrm{cm}^{2}$ & $\begin{array}{l}\text { Methylobacterium } \\
\text { extorquens }\end{array}$ \\
\hline $\begin{array}{l}\text { FHO28 - } \\
\text { Flex hose } \\
\text { Center }\end{array}$ & 2 & $\mathrm{CFU} / \mathrm{cm}^{2}$ & $\begin{array}{l}\text { Ralstonia } \\
\text { eutrophalpaucula } \\
\text { (nit +) }\end{array}$ \\
\hline $\begin{array}{l}\text { FH028- } \\
\text { Flex hose } \\
\text { Center }\end{array}$ & 1 & $\mathrm{CFU} / \mathrm{cm}^{2}$ & $\begin{array}{l}\text { Methylobacterium } \\
\text { extorquens }\end{array}$ \\
\hline $\begin{array}{l}\text { FH028- } \\
\text { Flex hose } \\
\text { Center }\end{array}$ & 1 & $\mathrm{CFU} / \mathrm{cm}^{2}$ & $\begin{array}{l}\text { Bradyrhizobium } \\
\text { japonicum }\end{array}$ \\
\hline $\begin{array}{l}\text { FH036- } \\
\text { Flex hose } \\
\text { Outlet }\end{array}$ & 5 & $\mathrm{CFU} / \mathrm{cm}^{2}$ & $\begin{array}{l}\text { Unidentified non- } \\
\text { fermenting GNR } \\
\text { (nit -) }\end{array}$ \\
\hline $\begin{array}{l}\text { FH036- } \\
\text { Flex hose } \\
\text { Outlet }\end{array}$ & 2 & $\mathrm{CFU} / \mathrm{cm}^{2}$ & $\begin{array}{l}\text { Raistonia } \\
\text { eutrophalpaucula } \\
\text { (nit }+ \text { ) }\end{array}$ \\
\hline $\begin{array}{l}\text { FH036- } \\
\text { Flex hose } \\
\text { Outlet }\end{array}$ & 2 & $\mathrm{CFU} / \mathrm{cm}^{2}$ & $\begin{array}{l}\text { Methylobacterium } \\
\text { extorquens }\end{array}$ \\
\hline
\end{tabular}

Seven-day enumeration results reported for surfaces of the male quick disconnect (QD) components included the spring poppet interface (FH006) with $32 \mathrm{CFU} / \mathrm{swab}$, webbing of poppet (FH007) with $3 \mathrm{CFU} / \mathrm{swab}$, and the surface of the exposed poppet (FH008) with 107 CFU/swab. The colony forming units per unit area sampled for other male QD surfaces was estimated from engineering drawing measurements including the poppet o-ring (FH009) at $308 \mathrm{CFU} / \mathrm{cm}^{2}$, forward housing between primary and secondary seals (FH011) at 535 $\mathrm{CFU} / \mathrm{cm}^{2}$, inside aft housing (FH013) at $6 \mathrm{CFU} / \mathrm{cm}^{2}$, and inside the hose barb (FH014) at $43 \mathrm{CFU} / \mathrm{cm}^{2}$. All of the isolates from these male QD samples were identified as nitrate positive Ralstonia eutrophalpaucula with some of the isolates producing nitrogen gas from nitrate.

Seven-day membrane filtration results for female QD surfaces included the hose barb (FH040) with 8 $\mathrm{CFU} / \mathrm{cm}^{2}$, back end of flow path (FHO42) with 3 CFU $/ \mathrm{cm}^{2}, 180^{\circ}$ forward part of sleeve stop (FH043) with $49 \mathrm{CFU} / \mathrm{cm}^{2}$, and outside the spring above the sleeve (FH045) with $7 \mathrm{CFU} / \mathrm{cm}^{2}$. The 14-day count for the inside housing of the wetted side of the o-ring was 15 $\mathrm{CFU} / \mathrm{cm}^{2}$. The isolate identifications for female $\mathrm{QD}$ components are shown in Table 2.

\section{Table 2 Isolate Identifications for Female QD Surfaces}

\begin{tabular}{|l|r|l|}
\hline \multicolumn{1}{|c|}{$\begin{array}{c}\text { Sample } \\
\text { Description }\end{array}$} & $\begin{array}{l}\text { Results } \\
\text { CFU/cm }\end{array}$ & Species Identification \\
\hline $\begin{array}{l}\text { FH040 - Barb } \\
1 / 2 \text { " From } \\
\text { Couple End }\end{array}$ & 8 & $\begin{array}{l}\text { Acidovorax species } \\
\text { (nit +) }\end{array}$ \\
\hline $\begin{array}{l}\text { FH042 - Back } \\
\text { End }\end{array}$ & 2 & $\begin{array}{l}\text { Methylobacterium } \\
\text { extorquens }\end{array}$ \\
\hline $\begin{array}{l}\text { FH042 - Back } \\
\text { End }\end{array}$ & 1 & $\begin{array}{l}\text { Ralstonia } \\
\text { eutrophalpaucula (nit -) }\end{array}$ \\
\hline $\begin{array}{l}\text { FH043 - 180 } \\
\text { Fwd Part of } \\
\text { Sleeve Stop }\end{array}$ & 49 & $\begin{array}{l}\text { Ralstonia } \\
\text { eutrophalpaucula (nit -) }\end{array}$ \\
\hline $\begin{array}{l}\text { FH045 - Spring } \\
\text { Above the } \\
\text { Sleeve }\end{array}$ & 3 & Bacillus atrophaeus \\
\hline $\begin{array}{l}\text { FH045 - Spring } \\
\text { Above the } \\
\text { Sleeve }\end{array}$ & 1 & $\begin{array}{l}\text { Ralstonia } \\
\text { eutrophalpaucula (nit - ) }\end{array}$ \\
\hline $\begin{array}{l}\text { FH045 - Spring } \\
\text { Above the } \\
\text { Sleeve }\end{array}$ & 1 & $\begin{array}{l}\text { Unidentified non- } \\
\text { fermenting GNR (nit -) }\end{array}$ \\
\hline $\begin{array}{l}\text { FH045 - Spring } \\
\text { Above the } \\
\text { Sleeve }\end{array}$ & 1 & Microbacterium species \\
\hline $\begin{array}{l}\text { FH045 - Spring } \\
\text { Above the } \\
\text { Sleeve }\end{array}$ & 1 & $\begin{array}{l}\text { Mycobacterium } \\
\text { mucogenicum }\end{array}$ \\
\hline $\begin{array}{l}\text { FH047 - Wetted } \\
\text { Side of O-Ring }\end{array}$ & 10 & $\begin{array}{l}\text { Lampropedia hyalina } \\
\text { (nit-) }\end{array}$ \\
\hline $\begin{array}{l}\text { FH047 - Wetted } \\
\text { Side of O-Ring }\end{array}$ & 5 & $\begin{array}{l}\text { Unidentified non- } \\
\text { fermenting GNR }\end{array}$ \\
\hline
\end{tabular}

Coolant removed from the IATCS channel of the SPCU heat exchanger (HX003) contained 4.40E+06 CFU/100 $\mathrm{ml}$ of bacteria (7-day incubation). The EMU Channel 1 fluid count at 7-days was $1.15 \mathrm{E}+04 \mathrm{CFU} / 100 \mathrm{ml}$. And 
the EMU channel 2 fluid contained $3.50 \mathrm{E}+03 \mathrm{CFU} / 100$ $\mathrm{ml}$ of bacteria (7-day incubation). The isolate identifications for SPCU heat exchanger fluids are provided in Table 3.

Table 3 Isolate Identifications for SPCU Heat Exchanger Fluids

\begin{tabular}{|c|c|c|}
\hline $\begin{array}{c}\text { Sample } \\
\text { Description }\end{array}$ & $\begin{array}{l}\text { Results } \\
\text { CFU/100 ML }\end{array}$ & Species Identification \\
\hline $\begin{array}{l}\text { HX003- } \\
\text { ITCS Fluid }\end{array}$ & $1.87 E+06$ & $\begin{array}{l}\text { Ralstonia } \\
\text { eutrophalpaucula (nit -) }\end{array}$ \\
\hline $\begin{array}{l}\text { HX003- } \\
\text { ITCS Fluid }\end{array}$ & $1.78 E+06$ & $\begin{array}{l}\text { Ralstonia } \\
\text { eutrophalpaucula (nit +) }\end{array}$ \\
\hline $\begin{array}{l}\text { HX003- } \\
\text { ITCS Fluid }\end{array}$ & $7.50 \mathrm{E}+05$ & $\begin{array}{l}\text { Acidovorax species } \\
\text { (produces ammonia } \\
\text { from nitrate) }\end{array}$ \\
\hline $\begin{array}{l}\text { HX003- } \\
\text { ITCS Fluid }\end{array}$ & $1.45 \mathrm{E}+03$ & $\begin{array}{l}\text { Aspergillus species } \\
\text { (fungi) }\end{array}$ \\
\hline $\begin{array}{l}\text { HX004- EMU } \\
\text { Channel } 1 \\
\text { Fluid }\end{array}$ & $5.75 E+03$ & $\begin{array}{l}\text { Acidovorax species } \\
\text { (nit +) }\end{array}$ \\
\hline $\begin{array}{l}\text { HX004- EMU } \\
\text { Channel } 1 \\
\text { Fluid - } 71.73 \\
\mathrm{ml}\end{array}$ & $2.88 \mathrm{E}+03$ & $\begin{array}{l}\text { Methylobacterium } \\
\text { extorquens }\end{array}$ \\
\hline $\begin{array}{l}\text { HX004 - EMU } \\
\text { Channel } 1 \\
\text { Fluid }\end{array}$ & $2.88 E+03$ & $\begin{array}{l}\text { Sphingomonas } \\
\text { parapaucimobilis }\end{array}$ \\
\hline $\begin{array}{l}\text { HX004 - EMU } \\
\text { Channel } 1 \\
\text { Fluid }\end{array}$ & $1.90 \mathrm{E}+03$ & $\begin{array}{l}\text { Aspergillus species } \\
\text { (fungi) }\end{array}$ \\
\hline $\begin{array}{l}\text { HX005- EMU } \\
\text { Channel } 2 \\
\text { Fluid }\end{array}$ & $3.50 E+03$ & $\begin{array}{l}\text { Ralstonia } \\
\text { eutrophalpaucula } \\
\text { (produces } \mathrm{N}_{2} \text { from } \\
\text { nitrate) }\end{array}$ \\
\hline
\end{tabular}

The enumeration results for surfaces from the IATCS channel of the SPCU heat exchanger are shown in Table 4.
Table 4 Membrane Filtration Enumeration Results for SPCU IATCS Channel

\begin{tabular}{|c|c|c|}
\hline Sample Description & $\begin{array}{c}\text { Incubation } \\
\text { Time }\end{array}$ & $\begin{array}{l}\text { Results } \\
\text { CFU/cm² }\end{array}$ \\
\hline $\begin{array}{l}\text { HX007 - Inlet before } \\
\text { Mạnifold Removal }\end{array}$ & 14-Day & 38 \\
\hline $\begin{array}{l}\text { HX009 - ITCS Outlet Swab } \\
\text { before Manifold Removal }\end{array}$ & 7-Day & $3.02 E+02$ \\
\hline $\begin{array}{l}\text { HX015 - Outlet End of Inlet } \\
\text { Manifold after Removal }\end{array}$ & 14-Day & 11 \\
\hline $\begin{array}{l}\text { HX019 - Outer Rim of Inlet } \\
\text { Manifold after Removal }\end{array}$ & 14-Day & $2.92 E+04$ \\
\hline $\begin{array}{l}\text { HX021 - 1/2 of Inlet Side } \\
\text { Face after manifold removal }\end{array}$ & 14-Day & $7.28 \mathrm{E}+02$ \\
\hline $\begin{array}{l}\text { HX022 - Outer End of } \\
\text { Outlet Manifold after } \\
\text { Manifold Removal }\end{array}$ & 7-Day & $<1$ \\
\hline $\begin{array}{l}\text { HX025- Outer Rim of Outlet } \\
\text { Manifold after Removal }\end{array}$ & 14-Day & $4.78 \mathrm{E}+03$ \\
\hline $\begin{array}{l}\text { HX027 - 1/2 ITCS Outlet } \\
\text { Side Face of Heat } \\
\text { Exchanger after Removal }\end{array}$ & 14-Day & 88 \\
\hline
\end{tabular}

The isolate identifications from the IATCS channel of SPCU heat exchanger are listed in Table 5. Identifications of a couple of different colony morphologies from a dilution that contained too many colonies to count were also performed and the concentration is reported as not available (NA).

\section{Table 5 Isolate Identifications for SPCU IATCS Channel Surfaces}

\begin{tabular}{|l|r|l|}
\hline \multicolumn{1}{|c|}{$\begin{array}{c}\text { Sample } \\
\text { Description }\end{array}$} & $\begin{array}{c}\text { Results } \\
\text { CFU/CM }\end{array}$ & \multicolumn{1}{|c|}{$\begin{array}{c}\text { Species } \\
\text { Identification }\end{array}$} \\
\hline $\begin{array}{l}\text { HX007 - Inlet before } \\
\text { Manifold Removal }\end{array}$ & 16 & $\begin{array}{l}\text { Methylobacterium } \\
\text { extorquens }\end{array}$ \\
\hline $\begin{array}{l}\text { HX007 - Inlet before } \\
\text { Manifold Removal }\end{array}$ & 15 & $\begin{array}{l}\text { Unid. non- } \\
\text { fermenting GNR (nit } \\
- \text { ) }\end{array}$ \\
\hline $\begin{array}{l}\text { HX007 - ITCS Inlet } \\
\text { before Manifold } \\
\text { Removal }\end{array}$ & 7 & $\begin{array}{l}\text { Unidentified non- } \\
\text { fermenting GNR (nit } \\
-)\end{array}$ \\
\hline
\end{tabular}


Table 5 Isolate Identifications for SPCU IATCS Channel Surfaces (continued)

\begin{tabular}{|c|c|c|}
\hline $\begin{array}{c}\text { Sample } \\
\text { Description }\end{array}$ & $\begin{array}{l}\text { Results } \\
\text { CFU/CM }\end{array}$ & $\begin{array}{c}\text { Species } \\
\text { Identification }\end{array}$ \\
\hline $\begin{array}{l}\text { HX009 - Outlet Swab } \\
\text { before Manifold } \\
\text { Removal }\end{array}$ & $2.20 \mathrm{E}+02$ & $\begin{array}{l}\text { Methylobacterium } \\
\text { extorquens }\end{array}$ \\
\hline $\begin{array}{l}\text { HX009 - Outlet Swab } \\
\text { before Manifold } \\
\text { Removal }\end{array}$ & 82 & $\begin{array}{l}\text { Lampropedia } \\
\text { hyalina (nit -) }\end{array}$ \\
\hline $\begin{array}{l}\text { HX015 - Outlet End } \\
\text { of Inlet Manifold after } \\
\text { Removal }\end{array}$ & 9 & $\begin{array}{l}\text { Unid. non- } \\
\text { fermenting GNR (nit } \\
- \text { ) }\end{array}$ \\
\hline $\begin{array}{l}\text { HX015 - Outlet End } \\
\text { of Inlet Manifold after } \\
\text { Removal }\end{array}$ & 1 & $\begin{array}{l}\text { Methylobacterium } \\
\text { extorquens }\end{array}$ \\
\hline $\begin{array}{l}\text { HX015 - Outlet End } \\
\text { of Inlet Manifold after } \\
\text { Removal }\end{array}$ & 1 & Bacillus atrophaeus \\
\hline $\begin{array}{l}\text { HX019 - Outer Rim } \\
\text { of Inlet Manifold after } \\
\text { Removal }\end{array}$ & $2.03 E+04$ & $\begin{array}{l}\text { Acidovorax species } \\
\text { (nit +) }\end{array}$ \\
\hline $\begin{array}{l}\text { HX019 - Outer Rim } \\
\text { of Inlet Manifold after } \\
\text { Removal }\end{array}$ & $5.40 E+03$ & $\begin{array}{l}\text { Methylobacterium } \\
\text { extorquens }\end{array}$ \\
\hline $\begin{array}{l}\text { HX019 - Outer Rim } \\
\text { of Inlet Manifold after } \\
\text { Removal }\end{array}$ & $3.50 E+03$ & $\begin{array}{l}\text { Unidentified non- } \\
\text { fermenting GNR } \\
\text { (nit -) }\end{array}$ \\
\hline $\begin{array}{l}\text { HX019 - Outer Rim } \\
\text { of Inlet Manifold after } \\
\text { Removal }\end{array}$ & $1.10 \mathrm{E}+03$ & $\begin{array}{l}\text { Sphingomonas } \\
\text { parapaucimobilis }\end{array}$ \\
\hline $\begin{array}{l}\mathrm{HX019} \text { - Outer Rim } \\
\text { of Inlet Manifold after } \\
\text { Removal }\end{array}$ & NA & Acidovorax facilis \\
\hline
\end{tabular}

The enumeration results for surfaces from EMU channel 1 and 2 of the SPCU heat exchanger are shown in Table 6.
Table 6 Membrane Filtration Enumeration Results for SPCU EMU Channels

\begin{tabular}{|l|c|r|}
\hline \multicolumn{1}{|c|}{ Sample Description } & $\begin{array}{c}\text { Incubation } \\
\text { Time }\end{array}$ & $\begin{array}{r}\text { Results } \\
\text { CFU/CM }\end{array}$ \\
\hline $\begin{array}{l}\text { HX013 - EMU 1 Outlet } \\
\text { before Manifold Removal }\end{array}$ & 7-Day & $1.13 E+02$ \\
\hline $\begin{array}{l}\text { HX014 - EMU 2 Inlet before } \\
\text { Manifold Removal }\end{array}$ & 7-Day & $1.33 E+02$ \\
\hline $\begin{array}{l}\text { HX030 - EMU 1 Inlet } \\
\text { Finstock Area after removal }\end{array}$ & 7-Day & $<12$ \\
\hline $\begin{array}{l}\text { HX032 - EMU 1 Inlet } \\
\text { Finstock Upper right Area } \\
\text { after Removal }\end{array}$ & 7-Day & 26 \\
\hline $\begin{array}{l}\text { HX034 - EMU 1 Inlet } \\
\text { Finstock Area, Lower Half } \\
\text { after Removal }\end{array}$ & 14-Day & 68 \\
\hline $\begin{array}{l}\text { HX036 - EMU 2 Inlet } \\
\text { Manifold Finstock Left Half } \\
\text { after Removal }\end{array}$ & 14-Day & $1.06 E+02$ \\
\hline $\begin{array}{l}\text { HX038 - EMU 1 Outlet } \\
\text { Manifold Finstock after } \\
\text { Manifold Removal }\end{array}$ & 14-Day & $2.13 E+02$ \\
\hline $\begin{array}{l}\text { HX041 - EMU 2 Outlet } \\
\text { Manifold Finstock after } \\
\text { Manifold Removal }\end{array}$ & 14-Day & 11 \\
\hline
\end{tabular}

The isolate identifications from EMU channel 1 and 2 of the SPCU heat exchanger are provided in Table 7.

Table 7 Isolate Identifications for SPCU EMU Channel Surfaces

\begin{tabular}{|l|r|l|}
\hline \multicolumn{1}{|c|}{$\begin{array}{c}\text { Sample } \\
\text { Description }\end{array}$} & $\begin{array}{c}\text { Results } \\
\text { CFU/CM }\end{array}$ & $\begin{array}{c}\text { Species } \\
\text { Identification }\end{array}$ \\
\hline $\begin{array}{l}\text { HX013 - EMU 1 } \\
\text { Outlet before } \\
\text { Manifold Removal }\end{array}$ & $1.13 E+02$ & $\begin{array}{l}\text { Ralstonia } \\
\text { eutrophalpaucula } \\
\text { (produces N } \\
\text { nitrate) }\end{array}$ \\
\hline $\begin{array}{l}\text { HX013 - EMU 1 } \\
\text { Outlet before } \\
\text { Manifold Removal }\end{array}$ & 16 & $\begin{array}{l}\text { Aspergillus species } \\
\text { (fungi) }\end{array}$ \\
\hline $\begin{array}{l}\text { HX013 - EMU 1 } \\
\text { Outlet before } \\
\text { Manifold Removal }\end{array}$ & 5 & $\begin{array}{l}\text { Aspergillus species } \\
\text { (fungi, different } \\
\text { colony morphology) }\end{array}$ \\
\hline
\end{tabular}


Table 7 Isolate Identifications for SPCU EMU Channel Surfaces (continued)

\begin{tabular}{|c|c|c|}
\hline $\begin{array}{c}\text { Sample } \\
\text { Description }\end{array}$ & $\begin{array}{l}\text { Results } \\
\text { CFU/CM }\end{array}$ & $\begin{array}{c}\text { Species } \\
\text { Identification }\end{array}$ \\
\hline $\begin{array}{l}\text { HX014 - EMU } 2 \\
\text { Inlet before } \\
\text { Manifold Removal }\end{array}$ & $1.21 \mathrm{E}+02$ & $\begin{array}{l}\text { Methylobacterium } \\
\text { extorquens }\end{array}$ \\
\hline $\begin{array}{l}\text { HX014 - EMU } 2 \\
\text { Inlet before } \\
\text { Manifold Removal }\end{array}$ & 12 & $\begin{array}{l}\text { Ralstonia } \\
\text { eutrophalpaucula }\end{array}$ \\
\hline $\begin{array}{l}\text { HX014 - EMU } 2 \\
\text { Inlet before } \\
\text { Manifold Removal }\end{array}$ & 11 & $\begin{array}{l}\text { Aspergillus species } \\
\text { (fungi) }\end{array}$ \\
\hline $\begin{array}{l}\text { HX032 - EMU } 1 \\
\text { Inlet Finstock }\end{array}$ & 13 & $\begin{array}{l}\text { Methylobacterium } \\
\text { extorquens (non- } \\
\text { pigmented) }\end{array}$ \\
\hline $\begin{array}{l}\text { HX032- EMU } 1 \\
\text { Inlet Finstock }\end{array}$ & 13 & $\begin{array}{l}\text { Bacillus megaterium! } \\
\text { simplex }\end{array}$ \\
\hline $\begin{array}{l}\text { HX034 - EMU } 1 \\
\text { Inlet Finstock Area }\end{array}$ & 64 & $\begin{array}{l}\text { Unidentified non- } \\
\text { fermenting GNR (nit } \\
+ \text { ) }\end{array}$ \\
\hline $\begin{array}{l}\text { HX034 - EMU } 1 \\
\text { Inlet Finstock Area }\end{array}$ & 64 & $\begin{array}{l}\text { Unidentified non- } \\
\text { fermenting GNR (nit } \\
+ \text { ) }\end{array}$ \\
\hline $\begin{array}{l}\text { HX034- EMU } 1 \\
\text { Inlet Finstock Area }\end{array}$ & 2 & $\begin{array}{l}\text { Bacillus megaterium/ } \\
\text { simplex }\end{array}$ \\
\hline $\begin{array}{l}\text { HX034 - EMU 1 } \\
\text { Inlet Finstock Area }\end{array}$ & 2 & Bacillus filicolonicus \\
\hline $\begin{array}{l}\text { HX036 - EMU } 2 \\
\text { Inlet Manifold } \\
\text { Finstock }\end{array}$ & 77 & $\begin{array}{l}\text { Unidentified non- } \\
\text { fermenting GNR (nit } \\
+ \text { ) }\end{array}$ \\
\hline $\begin{array}{l}\text { HX036 - EMU } 2 \\
\text { Inlet Manifold } \\
\text { Finstock }\end{array}$ & 27 & $\begin{array}{l}\text { Methylobacterium } \\
\text { mesophilicum/ } \\
\text { radiotolerans }\end{array}$ \\
\hline $\begin{array}{l}\text { HX036- EMU } 2 \\
\text { Inlet Manifold } \\
\text { Finstock }\end{array}$ & 2 & Bacillus atrophaeus \\
\hline $\begin{array}{l}\text { HX036 - EMU } 2 \\
\text { Inlet Manifold } \\
\text { Finstock }\end{array}$ & 2 & $\begin{array}{l}\text { Aspergillus species } \\
\text { (fungi) }\end{array}$ \\
\hline $\begin{array}{l}\text { HX038 - EMU } 1 \\
\text { Outlet Manifold } \\
\text { Finstock }\end{array}$ & $2.08 \mathrm{E}+02$ & $\begin{array}{l}\text { Unidentified non- } \\
\text { fermenting GNR (nit } \\
+ \text { ) }\end{array}$ \\
\hline $\begin{array}{l}\text { HX038 - EMU } 1 \\
\text { Outlet Manifold } \\
\text { Finstock }\end{array}$ & 5 & $\begin{array}{l}\text { Unidentified non- } \\
\text { fermenting GNR (nit } \\
+ \text { ) }\end{array}$ \\
\hline $\begin{array}{l}\text { HX041 - EMU } 2 \\
\text { Outlet Manifold }\end{array}$ & 11 & $\begin{array}{l}\text { Unidentified non- } \\
\text { fermenting GNR (nit }\end{array}$ \\
\hline
\end{tabular}

\begin{tabular}{|l|l|l|}
\hline Finstock & & + ) \\
\hline
\end{tabular}

SUMMARY AND CONCLUSIONS

The level of planktonic microorganisms in IATCS coolant samples returned from the on-orbit has remained stable between 500,000 to 1 million colony forming units per $100 \mathrm{ml}$ over the past three years verified by analysis of coolant samples returned on the Shuttle and Soyuz. The levels of heterotrophic microorganisms in the fluid removed from the flex hose assembly and the IATCS channel in the SPCU heat exchanger were consistent with the historical data. Microbiological concentrations ranged from 1 to 2 logs lower in the water samples removed from the EMU channels in the SPCU heat exchanger. The lower microbiological levels may reflect a combination of the effect of periodic iodination to control bacterial growth, different concentrations and types of nutrients, and stagnant conditions from infrequent operations required for EVAs.

Historically, microbial growth in water systems has created problems for water circulation equipment and system functionality. Almost all known bacterial species are capable of extended survival in aquatic systems, and are capable of a wide range of metabolic activity even in water with extremely low nutrient content $(6,7)$. Biofilms readily form on exposed surfaces of almost any material in a flowing water system (Costerton et al., 1985; Willis and Schultz, 1987; Lappin-Scott and Costerton, 1989). These biofilms may actually represent a majority of the microbial biomass in a low nutrient system $(8,9,10)$. However, the surface sample microbiological enumerations from the flex hose assembly, associated QDs, and the SPCU heat exchanger were significantly lower than anticipated. The levels are indicative of the presence of individual cells and a few microcolonies on most surfaces. Most surfaces had microbiological counts less than $100 \mathrm{CFU} / \mathrm{cm}^{2}$ and the counts for the flex hose and IATCS passages in the SPCU heat exchanger were less than $10 \mathrm{CFU} / \mathrm{cm}^{2}$. Concentrations of bacteria greater than $100 \mathrm{CFU} / \mathrm{cm}^{2}$ were found on the male QD poppet, o-rings, and between primary and secondary seals, and the SPCU manifolds and welds. The data indicates that microorganisms are not causing any biofouling, significant reductions in flow rates, or reduction of heat transfer in the flex hose assembly and SPCU heat exchanger.

The selection of nickel braze materials as a coating for the stainless steel parting sheets in the IATCS heat exchangers and cold plates may be involved in the inhibition of surface attachment and proliferation of bacterial cells. The passive film that forms on nickel in alkaline borate buffer at ph 8.4 was determined by in situ surface enhanced Raman spectroscopy to be a single species consisting of amorphous/microcrystalline $\mathrm{Ni}(\mathrm{OH})_{2}(11)$. The leading hypothesis is that periodic sloughing of the weakly adherent $\mathrm{Ni}(\mathrm{OH})_{2}$ layer prevents permanent attachment of bacteria and significant biofilm 
formation. A review of biofouling studies on cupronickel materials have shown that the weakly adherent, porous passive layers formed during secondary corrosion reactions prevents permanent adhesion of microorganisms (12). The same phenomenon may also be preventing biofilm formation on the Teflon flex hose due to the prevalence of loosely associated nickel precipitates.

The predominate bacterial species identified in the IATCS coolant, EMU water, and surfaces on the flex hose, QDs, and SPCU heat exchanger are aerobic, motile, Gram negative, non-fermentative, heterotrophs that grow well on organic acids, amino acids, peptone, and limited carbohydrates. Some of these bacteria are lithotrophs capable of oxidizing the molecular hydrogen produced at a galvanic cathode, thereby depolarizing it and increasing the rate of corrosion. These organisms are also autotrophs and are capable of obtaining all the carbon they need from inorganic sources such as carbon dioxide (13). Most of these organisms including Ralstonia species, Acidovorax species, Methylobacterium species, and Sphingomonas species are commonly found in deionized water systems. A few gram positive species including Bacillus and Microbacterium were also found in low numbers in the QDs and SPCU heat exchanger. These microorganisms may represent laboratory sampling and handling contamination even though good aseptic techniques were utilized due to the fact that these organisms were at low numbers and were found during the later phases of the destructive sampling. An Aspergillus species which is a filamentous fungus was isolated from SPCU fluids and surfaces. This may indicate contamination of the SPCU heat exchanger during storage on-board the ISS, presence of fungi in the caps that were placed on the inlets and outlets, or contamination during packing and shipping from the ISS.

In conclusion, surface phenomena associated with nickel hydroxide and nickel phosphate precipitates appear to be preventing significant biofilm formation in the flex hose assembly and SPCU heat exchanger studied. It should be noted that the growth of microorganisms have not resulted in adverse system impacts such as loss of heat transfer or microbiologically influenced corrosion. Nominal IATCS operations have not indicated the requirement for immediate remediation of microorganisms. However, the stable planktonic population of microorganisms in the coolant between 500,000 and 1 million CFU/100 ml may indicate the presence of biofilms on other wetted materials in the system including stainless steel, titanium, ethylene propylene rubber, and epoxy resins. In the long-term, the implementation of a safe, effective, stable and compatible antimicrobial for the IATCS loops currently on-orbit and for future ISS elements and payloads is recommended. The addition of an effective antimicrobial to the IATCS fluid will provide microbial control and minimize microbiological-related problems in the future. 


\section{ACKNOWLEDGEMENTS}

The research summarized in this paper was conducted under Contract NAS15-10000 for the NASA International Space Station Program. The technical assistance provided by Tyler Anderson, Susan Broadaway, Sharon Chang Abby Guilbert, Sarah Roth, Kerry Williamson, at Montana State University, is gratefully acknowledged.

\section{REFERENCES}

1. Clesceri, L.S., Greenberg, A.E., and Trussell, R.R. 1989. Standard Methods for the Examination of Water and Wastewater, 17th Ed. American Public Health Association, Washington, DC.

2. Holt, J.G., Krieg, N.R., Sneath, P.H.A., Staley, J.T. and Williams, S.T. Bergey's Manual of Determinative Bacteriology. Ninth Edition. 1994. Williams \& Wilkins, Baltimore.

3. MIDI Company Website: www.midi-inc.com.

4. Biolog Company Website: www.biolog.com.

5. Qualicon Company Website: www.qualicon.com.

6. Hamilton, W.A. 1987. Biofilms: Microbial Interactions and Metabolic Activities, p. 361-385. In T.R.G. Gray and J.G. Jones (ed.), Ecology of Microbial Communities. Cambridge University Press, Cambridge.

7. Hamilton, W.A. and Characklis, W.G. 1989. Relative Activities of Cells in Suspension and in Biofilms. $p$. 1992-1998. In W.G. Characklis and P.A. Wilderer (ed.), Structure and Function of Biofilms. John Wiley \& Sons, Inc. New York.

8. Costerton, J.W., Marrie T.J., and Cheng, K.J. 1985. Phenomena of Bacterial Adhesion, p. 3-43. In D.C. Savage and M. Fletcher (ed.), Bacterial Adhesion. Plenum Publishing Corp., New York.

9. Willis, C.E. and Schultz, J.R. 1987. Spacecraft Water System Disinfection Technology: Past, Present, and Future Needs. 17th Intersociety Conf. Environ. Sys., Seattle, WA, SAE Paper No. 871487.

10. Lappin-Scott, H.L. and Costerton, W.J. 1989. Bacterial Biofilms and Surface Fouling. Biofouling 1:323-342.

11. Oblonsky, L.J. and Devine, T.M. 1995. A Surface Enhanced Raman Spectroscopic Study on the Passive Films Formed in Borate Buffer on Iron, Nickel, Chromium and Stainless Steel. Corrosion Science, Vol.37, No.1, pp. 17-41.

12. Schleich, W. 2005. Application of Copper-Nickel Alloy UNS C70600 for Seawater Service. KM Europa Metal AG, Klosterstr. 29.

13. Moat, A.G. 1979. Microbial Physiology. John Wiley \& Sons, New York.

\section{ACRONYMS}

Internal Active Thermal Control System - IATCS External Active Thermal Control System- EATCS International Space Station - ISS

Thermal Control System - TCS

Special Performance Checkout Unit - SPCU

Extravehicular Mobility Units - EMUs

Extravehicular Activity - EVA

Heat Exchanger - $\mathrm{HX}$

Colony Forming Units - CFU

Milliliters - ml

Ethylenediaminetetraacetic acid - EDTA

Microbial Identification System, Inc. - MIDI

Deoxyribonucleic Acid - DNA

Quick Disconnect - QD

Nitrate Reduction Test (Nitrate Reduced to Nitrite) - nit 